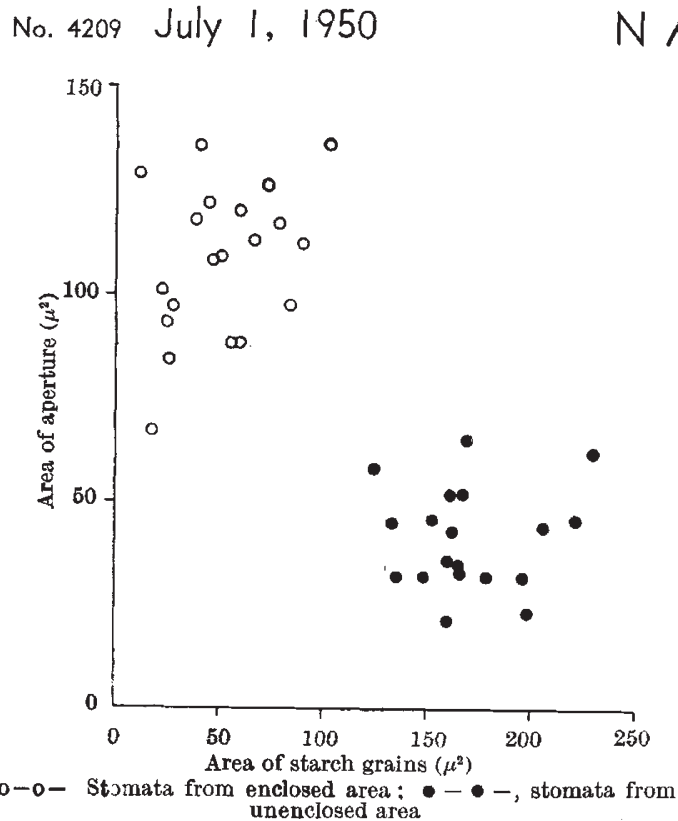

in one plane, and almost invariably some are slightly out of focus; this difficulty could be eliminated by direct projection of the original slide on to a screen for tracing, re-focusing as necessary. However, the necessary equipment has not been available, and the photographic method has therefore been adopted for preliminary experiments. The use of a photometric method for measuring the areas has been fully considered, but, for reasons which cannot be detailed here, rejected.

We have carried out two simple experiments which demonstrate the striking results that the technique affords. In the first, part of an illuminated Pelargonium leaf was enclosed between two glass plates; it is already known ${ }^{4,5,6}$ that the resulting drop in carbon dioxide concentration causes wide stomatal opening between the plates. A strip of lower epidermis was taken from both enclosed and unenclosed parts of the leaf by Lloyd's ${ }^{7}$ method, the strips were transferred to Heath's reagent, and the starch and aperture measured in twenty stornata from each strip. The results are shown in the accompanying scatterdiagram. It will be immediately clear that, within any one treatment, there is no sign whatever of the high negative correlation that classical theory might lead us to expect; but that enclosure has significantly increased aperture and reduced starch. It might still be argued that starch-sugar balance, though not responsible for variations in aperture between individual stomata under substantially constant conditions, yot controls major changes in aperture. However, Heath ${ }^{2}$, referring particularly to the work of Iljin', has pointed out that enclosure also increases the humidity of the air so enclosed, and has implied that there may be two effectsreduction of carbon dioxide acting upon aperture, increase of humidity acting upon starch-which have been confounded. Despite the intractability of Pelargonium in February, we have attempted to investigate this by enclosing part of a leaf between plates incorporating a drying-agent (calcium chloride). Visual inspection discloses immediately that such enclosure does not cause any appreciable diminution in starch-content; in fact, our computations suggest that there has been a statistically significant increase in both aperture and starch. However, since we are as yet ignorant of the extent to which these quantities vary over the surface of any one leaf, we place no reliance on this result; but it at least seems clear that the technique here outlined will enable us to undertake a fully quantitative investigation of this and related phenomena.

We are indebted to Dr. O. V. S. Heath for the Ioan of apparatus and to Mr. G. L. Robbins of Kodak, Ltd., for technical advice. A full discussion of the technique and a detailed account of the preliminary experiments will be published elsewhere in due course.

\section{W. T. WiLliams}

Botany Department

G. S. SPENCER

Bedford College for Women, London, N.W.1.

Feb. 22.

' Heath, O. V. S., Nature, 159, 647 (1947).

${ }^{2}$ Heath, O. V. S., New Phyt., 48, 186 (1949).

Small, J., et al., Proc. Roy. Soc. Edin., B, 61, 233 (1942)

Heath, O. V. S., and Williams, W. T., Nature, 161, 178 (1948).

- Heath, O. V. S., Nature, 161, 179 (1948).

- Williams, W. T., Ann. Bot., N.S., 18, 309 (1949).

Lloyd, F. E., Pub. Carneg. Inst., 82 (1908)

' Iljin, W. S., Beih. Bot. Zbl., 32, 15 (1914).

\section{High Light Intensities for Experiments in Crop Physiology}

Axtension has recently been devoted in this Department to the development of a growth cabinet in which the environment can be controlled, eventually for use in pasture research in New Zealand. For this purpose the critical requirement is high light intensity with the quality of daylight, over an area large enough for adequate replication of the experimental plants. It is the experience of plant physiologists that it is difficult to obtain such lighting without the complications caused by excessive radiation. The following note may therefore be of interest.

The growth cabinet used can be run at any temperature from $40^{\circ} \mathrm{F}$. to $75^{\circ} \mathrm{F}$. $\pm 2^{\circ} \mathrm{F}$., by circulating air past a heater and a thermostatically controlled cooler. There is no water screen between the lights and the plants. The evenly illuminated area is $5 \mathrm{sq}$. ft. ; it receives light from twelve 150 -watt tungsten filament reflector spotlamps and twelve experimental 30-watt, 3-ft., blue fluorescent tubes. These lamps provide a continuous spectrum which, compared with sunlight, is slightly weaker in green and stronger in blue and red. The present maximum intensity at the level of the plants, $18 \mathrm{in.}$ from the lamps, is equivalent to sunlight at 2,000 foot-candles. Below each tungsten bulb is a disk of Chance's 'O.N.20' heat-absorbing glass 6 in. in diameter. Even when more than two kilowatts are being put into the lamp circuit, it has been necessary to switch on an 80-watt heater to maintain cabinet temperature at $70^{\circ} \mathrm{F}$.

Experiments are being carried out. on strains of Lolium under this light intensity. The evidence to date does not suggest that 2,000 foot-candles is the maximum to which Lolium will respond, and arrangements are being made to increase the light intensity still further.

In the design of the lighting arrangements particular thanks are due to Dr. J. N. Aldington, of Siemens, Ltd.

Department of Botany,

University, Manchester.

K. J. Mitchel. May 16. 